\title{
Anterior insula activity predicts the influence of positively framed messages on decision making
}

\author{
Adam Krawitz, Rena Fukunaga, and Joshua W. Brown \\ Indiana University, Bloomington, Indiana
}

\begin{abstract}
The neural mechanisms underlying the influence of persuasive messages on decision making are largely unknown. We address this issue using event-related fMRI to investigate how informative messages alter risk appraisal during choice. Participants performed the Iowa Gambling Task while viewing a positively framed, negatively framed, or control message about the options. The right anterior insula correlated with improvement in choice behavior due to the positively framed but not the negatively framed message. With the positively framed message, there was increased activation proportional to message effectiveness when less-preferred options were chosen, consistent with a role in the prediction of adverse outcomes. In addition, the dorsomedial and the left dorsolateral prefrontal cortex correlated with overall decision quality, regardless of message type. The dorsomedial region mediated the relationship between the right anterior insula and decision quality with the positively framed messages. These findings suggest a network of frontal brain regions that integrate informative messages into the evaluation of options during decision making. Supplemental procedures and results for this article may be downloaded from http://cabn.psychonomic-journals.org/content/supplemental.
\end{abstract}

Persuasive messages are abundant in modern society and take a variety of forms, from public service announcements to political campaign slogans to product advertisements. These messages target different decisions, but they are all intended to influence the choices made by individuals. The question of how to persuade people to make more rational, adaptive decisions is an urgent one. The rise of cognitive and affective neuroscience suggests a new approach to the study of persuasion: Just as advancing our understanding of the molecular basis of pharmaceuticals has led to the development of more effective medicine, so too may advancing our understanding of the neural basis of persuasive messages lead to the development of more effective interventions for behavior change. To pursue this approach, we used the Iowa Gambling Task (IGT; Bechara, Damasio, Damasio, \& Anderson, 1994), with positively and negatively framed informative messages about the nature of the choice alternatives, in a rapid event-related fMRI design (see Figure 1). Our primary focus was on determining the brain areas that underlie the influence of informative messages on choice during decision making under uncertainty.

The influence of messages on choice behavior is a phenomenon distinct from that of decision making per se. The critical issue is that a message is an independent communication, separate from the presentation of choice alternatives. Although the neural basis of decision making has been studied extensively, relatively little is known about the brain regions involved specifically in the influence of informative messages on decision making. There have been recent studies of the effects of political messages (Kato et al., 2009), brand identity (McClure et al., 2004), and expert endorsement (Klucharev, Smidts, \& Fernández, 2008), but these studies have been focused on changes in attitudes rather than changes in choice behavior, and behavioral and stated preferences need not be correlated (McClure et al., 2004).

Although there is a paucity of functional-imaging data, behavioral work and supporting theory suggest that the effectiveness of persuasive messages depends, at least in part, on their alteration of the appraised risk of available options (Rothman \& Salovey, 1997). Furthermore, it is well established that risk appraisals during choice are important to human decision making. Two regions that may be key to risk appraisal are the anterior insula (AI) and the anterior cingulate cortex (ACC) (e.g., Behrens, Woolrich, Walton, \& Rushworth, 2007; Brown \& Braver, 2007; Fukui, Murai, Fukuyama, Hayashi, \& Hanakawa, 2005; Paulus \& Frank, 2006; Preuschoff, Quartz, \& Bossaerts, 2008).

The AI and the neighboring inferior frontal operculum (IFO) have typically been considered together as a single module involved in the representation of abstract and subjective feeling states (Craig, 2002). Recent proposals have suggested a significant role for affect in risk appraisal during decision making (Bechara \& Damasio, 2005; Loewenstein, Weber, Hsee, \& Welch, 2001). Activations have been identified in the AI during decision making (Lin, Chiu, Cheng, \& Hsieh, 2008) and for bad decisions compared with good decisions (Lawrence, Jol- 

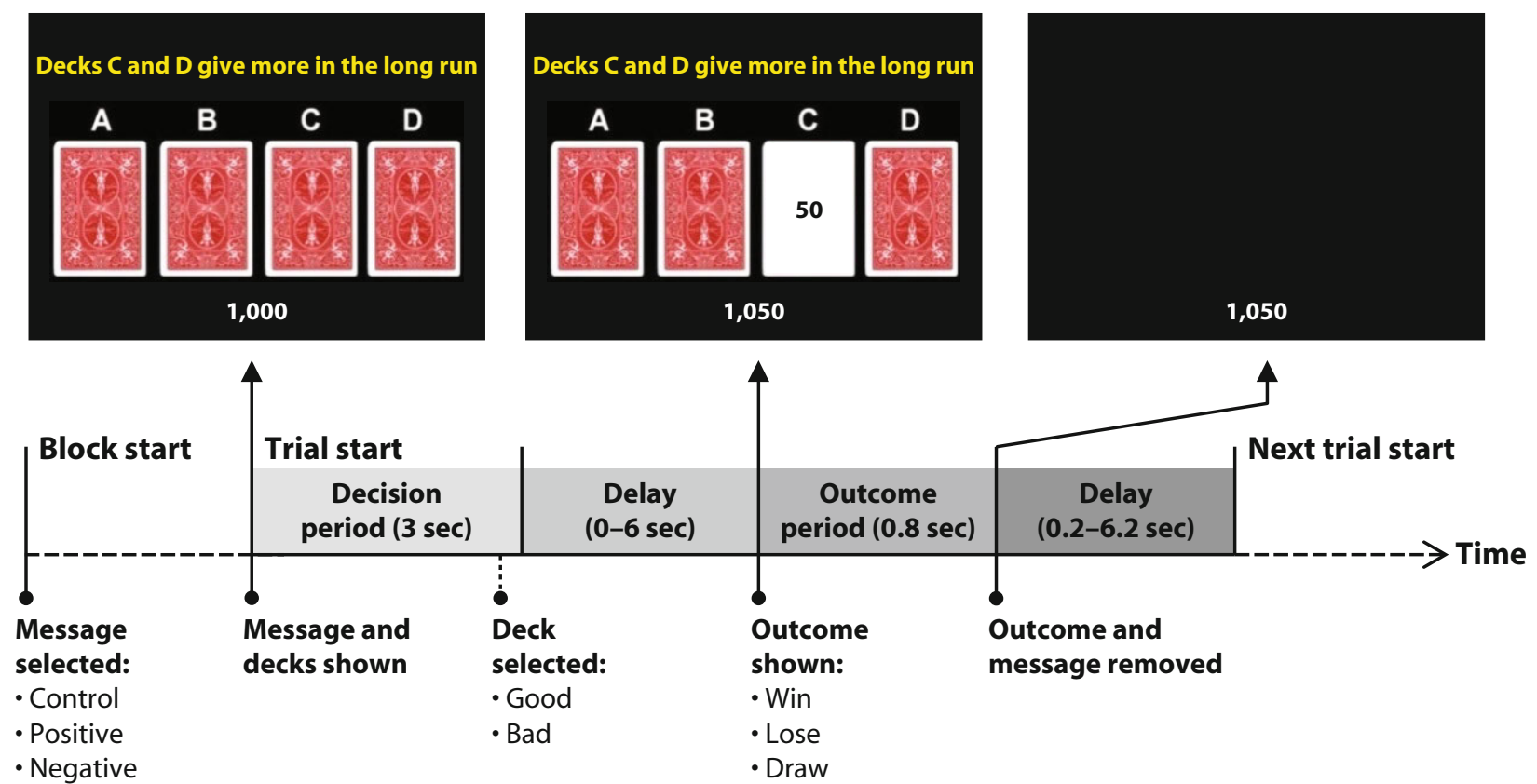

Figure 1. Schematic of a trial of the Iowa Gambling Task (Bechara, Damasio, Tranel, \& Damasio, 1997) with informative messages in a rapid event-related fMRI design. The images across the top represent the visual layout of the display at various times during the trial. The diagram at the bottom indicates trial time course with durations and relevant events as classified for fMRI analysis. This and all subsequent figures are in color in the online version of the article.

lant, O’Daly, Zelaya, \& Phillips, 2009), and AI activation has correlated with predicted risk and the error in those predictions (Preuschoff et al., 2008). In addition, greater AI activation precedes decisions to avoid risk (Kuhnen \& Knutson, 2005), and, in substance abusers, hypoactivity in the AI during decision making under uncertainty predicts later relapse (Paulus, Tapert, \& Schuckit, 2005).

In the ACC, as in the AI, activations have been identified during decision making (Lin et al., 2008) and for bad decisions compared with good decisions (Fukui et al., 2005; Lawrence et al., 2009). More generally, greater ACC activity correlates with more normative decision making (Paulus \& Frank, 2006), and performance-monitoring processes in the ACC are critical to learning the likelihood of an error (Brown \& Braver, 2005) and the potential consequences of risky behavior (Brown \& Braver, 2007, 2008). These findings are consistent with work implicating the ACC in predicting undesirable outcomes (Brown \& Braver, 2005, 2007) and driving risk aversion (Magno, Foxe, Molholm, Robertson, \& Garavan, 2006; Paulus \& Frank, 2006). In substance abusers, increased risk taking is associated with hypoactivity in the ACC (Fishbein et al., 2005); conversely, in obsessive-compulsive disorder, long-term hyperactivity of the ACC leads to inappropriate, excessive effort to avoid mistakes (Gehring, Himle, \& Nisenson, 2000).

To the extent that informative messages influence the appraised risk for a chosen option, this work collectively suggests that the AI and the ACC are likely places to observe these effects. Indeed, in both the AI (Paulus, Rogalsky, Simmons, Feinstein, \& Stein, 2003) and the ACC (Fukui et al., 2005), larger activations are correlated with the selection of risky relative to safe options, and the larger this difference is, the less likely participants are to choose the risky options. We refer to this difference in activation for risky relative to safe options as the risk effect. Also note that although the term risk anticipation has been used to describe the mental process represented by the risk effect (e.g., Fukui et al., 2005), we will use the term risk appraisal to emphasize that although potential outcomes are being anticipated, risk itself is being calculated. These findings lead us to hypothesize that the effectiveness of messages in improving choice behavior will be correlated with the increase in the risk effect in the AI and ACC in the presence of informative messages relative to in their absence.

If we do find evidence for modulation of the risk effect correlated with message effectiveness in the AI and ACC, a follow-up issue will be the relative roles of these two areas. The framework provided by Craig (2002) conceives of the AI as limbic sensory cortex feeding into the ACC as limbic motor cortex. Furthermore, the somatic marker hypothesis suggests that the AI is involved in the representation of how potential adverse outcomes would feel, whereas the ACC is involved in guiding decision making away from actions leading to those outcomes (Bechara \& Damasio, 2005). These ideas are consistent with known anatomical pathways from the AI to the ACC (Barbas \& Pandya, 1989). On the basis of this work, we hypothesize that modulations of risk appraisal due to informative messages in the AI are integrated into the decision-making process in the ACC. This hypothesis lends itself to evaluation with mediation analysis (Wager, Davidson, Hughes, Lindquist, \& Ochsner, 2008).

An important factor bearing on the effectiveness of messages in changing choice behavior is how they are 
framed (Rothman \& Salovey, 1997). The framing of messages is related to but distinct from the framing of options used by Tversky and Kahneman (1981) to reveal the classic framing effects. In message-framing manipulations, the participant already has a set of available options whose framing is not altered. Instead, messages are altered to emphasize positive or negative aspects of those options. Typically, certain options are preferred to others, and the framed messages are evaluated on the basis of their effectiveness in increasing selection of the preferred options (O'Keefe \& Jensen, 2007). These frames are commonly referred to either as positive and negative frames or gain and loss frames. We use the terms positive and negative to avoid confusion with the classic framing of options.

The mechanisms and influence of framing on the effectiveness of messages is an important, complex, and unresolved question in the field of health behavior change (O'Keefe \& Jensen, 2007). Although findings are equivocal about differences in the overall relative effectiveness of positively and negatively framed messages, the fact that differences have been found on the individual level suggests that framing may alter the underlying neural mechanisms even if it fails to be revealed in behavior at the population level. Intriguingly, gain versus loss framing of the options in a decision leads to differential ACC activity during decision making (De Martino, Kumaran, Seymour, \& Dolan, 2006). To the extent to which a cognitive neuroscience approach can provide new insight into the neural basis of message effectiveness, message framing is an important factor with well-defined applications for improving public health.

We use the IGT as the context for studying the effectiveness of positively and negatively framed messages. The IGT is a model of real-world decision making under uncertainty (Bechara, Damasio, Tranel, \& Damasio, 1997). Despite being criticized on various counts (Chiu \& Lin, 2007; Chiu et al., 2008; Dunn, Dalgleish, \& Lawrence, 2006; Lin, Chiu, Lee, \& Hsieh, 2007), the IGT has been used extensively to study and model decision making in both clinical (e.g., Bechara et al., 1994; Bechara et al., 2001) and nonclinical (e.g., Suhr \& Tsanadis, 2007; Wetzels, Vandekerckhove, Tuerlinckx, \& Wagenmakers, 2010) populations (for a review, see Vorhold, 2008) and in functional neuroimaging studies (Fukui et al., 2005; Lawrence et al., 2009; Lin et al., 2008; Northoff et al., 2006). We used temporal jittering to allow separate estimation of brain activation for decision making and for outcome evaluation (Dale, 1999), since our focus here is specifically on the time of choice. Activations related to decisions versus outcomes have been confounded in previous studies (e.g., Fukui et al., 2005; Lawrence et al., 2009).

In the IGT, initially naive participants repeatedly choose from one of four card decks, each with a different payoff schedule, and receive a monetary reward or punishment for each selection. The stated goal for the participants is to maximize total monetary reward. Two good decks have positive expected value, and two bad decks have negative expected value. However, the typical win is larger for the bad decks than for the good decks, putting potential im- mediate gains into opposition with long-term cumulative outcomes.

We refer to the bad decks as being riskier than the good decks. This designation of riskiness merits discussion, because the definition of risk is contested in the literature. In the economics and neuroeconomics literature, it generally refers to the variance of a known distribution (e.g., Knight, 1921; Preuschoff, Bossaerts, \& Quartz, 2006), but it often carries the competing meaning of the possibility of adverse outcomes in other literature, including the decision sciences and health psychology (e.g., Brown \& Braver, 2007, 2008; Rothman \& Salovey, 1997; Saaty, 1987; Yates, 1992). As an illustration of the distinction, an option could yield a wide range of possible monetary gains but no losses and could therefore entail risk in the sense of variance but no risk in the sense of possibility of loss.

The IGT decks vary along multiple dimensions of both probability and magnitude of reward and punishment (Chiu et al., 2008; Dunn et al., 2006). In particular, the bad decks are riskier whether risk is defined as the possibility of adverse outcomes or as variance. Specifically, although the probability of a loss is similar between the good and bad decks, the bad decks nevertheless entail both a higher magnitude of potential losses and a higher variance in the outcome payoffs. This allows us to investigate the neural correlates of message effectiveness during decision making under uncertainty without committing to a particular definition of risk.

\section{METHOD}

\section{Participants}

We recruited participants from the student body of Indiana University, Bloomington. They were required to be at least 18 years of age, to be right-handed, and to meet standard health and safety requirements for entry into the magnetic resonance imaging scanner. They were paid $\$ 25 / \mathrm{h}$ for participation, plus performance bonuses based on points earned during the task. Although 44 participants began the study, the data from 27 participants (mean age $=$ 22.1 years, age range $=18-28$ years, 13 female) were used in all of the reported analyses. Imaging was not initiated for 2 participants because they did not meet all of the participation and safety requirements, 2 elected to quit the study while it was in progress, data collection failed for 4 because of equipment malfunctions, 8 were excluded from the analysis because of transient spike artifacts in the imaging data caused by an equipment malfunction, and 1 was excluded because of a lack of trials in one cell of the design.

\section{Design and Procedure}

After receiving verbal instructions and completing separate individual difference measures for another study, the participants completed a few trials of the control condition of the IGT to become familiar with the task and the manner of response. During fMRI data collection, the participants performed the IGT for three blocks of 100 trials each. For each block, a different hint message was presented to the participants. The message was a control message ("Some decks are better than others"), a positively framed informative message ("Decks C and D give more in the long run"), or a negatively framed informative message ("Decks A and B give less in the long run"). The messages are qualitative, not quantitative in their informational content, but this also makes them more realistic, because persuasive messages in applied fields such as health communications are rarely quantifiable (Rothman \& Salovey, 1997). The order of the messages was counterbalanced across participants. 
During the task, there were four decks of cards, labeled A, B, C, and $\mathrm{D}$ from left to right. Two of the decks were considered bad decks: a net-loss/frequent-loss deck, with $50 \%$ loss trials, a mean loss of 25 points per trial, a gain of 100 on nonloss trials, and a variance of 18,125 ; and a net-loss/rare-loss deck, with $10 \%$ loss trials, a mean loss of 25 points per trial, a gain of 100 on nonloss trials, and a variance of 140,625 . The other two decks were considered good decks: a net-gain/frequent-loss deck, with $50 \%$ loss trials, a mean gain of 25 points per trial, a gain of 50 on nonloss trials, and a variance of 781; and a net-gain/rare-loss deck, with $10 \%$ loss trials, a mean gain of 25 points per trial, a gain of 50 on nonloss trials, and a variance of 5,625 . The bad decks were always adjacent, as were the good decks. For both the bad and the good decks, the frequent-loss deck was always to the left of the rare-loss deck. The order of bad and good decks was counterbalanced across blocks and participants.

The specific sequences of gains and losses for each deck were the same as in the original task design (Bechara et al., 1994). However, unlike in the original design, each trial outcome was presented as a net gain, draw, or loss; the participant started with an initial sum of 1,000 points; and the entire task was performed on a computer using E-Prime 1.2 (Psychology Software Tools, Pittsburgh, PA).

The participants were instructed to select cards from the decks. They were informed that the goal was to maximize earnings and that they would receive a monetary bonus based on the number of points that they accumulated. They were informed of the 3-sec period in which to make each selection and the running point total at the bottom of the screen. They were told that hint messages would appear at the top of the screen and that following these messages could lead to improved performance. They were told nothing about the order of the decks or how the order might change from block to block.

The timing and presentation of a trial is presented schematically in Figure 1. The participants' running point total was displayed throughout the block at the bottom of the screen. At the start of a trial, the current hint message and the four decks of cards were presented. The participants had $3 \mathrm{sec}$ to select a deck by pressing one of their middle or index fingers on buttons corresponding in a spatially compatible way to the decks. If the participants failed to respond within $3 \mathrm{sec}$, the trial was considered a no-response trial. After the response deadline, there was an exponentially distributed delay of $0,2,4$, or $6 \mathrm{sec}$. Following the variable delay, a card from the chosen deck was flipped over to reveal the outcome as a negative, zero, or positive point value, and the running total was updated. On no-response trials, the outcome was always a loss of 100 points, in order to encourage the participants to make a choice on every trial. The feedback remained visible for $0.8 \mathrm{sec}$, after which the message, cards, and outcome were removed for an exponentially distributed intertrial interval of $0.2,2.2,4.2$, or $6.2 \mathrm{sec}$ before the next trial began. The variable-length delays between choice and outcome and between trials were designed to allow the brain activity associated with the decision period to be estimated separately from that associated with response to the outcome (Dale, 1999).

\section{Scoring}

Decision quality is defined as the normalized difference in the number of selections from good decks and bad decks, with -1 indicating all bad deck selections, 1 indicating all good deck selections, and 0 indicating an equal number of selections from bad and good decks. To compare performance with a framed informative message and with the control message, message effectiveness is defined as the difference in decision quality between trial blocks. Decision quality and message effectiveness are robust against variance in the total number of decisions across blocks and participants and provide a relative measure of the numbers of good and bad decisions independent of the actual points earned.

\section{fMRI Analysis}

Image acquisition and preprocessing. Imaging data were collected on a 3.0 Tesla Siemens Magnetom Trio. For each participant, functional BOLD data were collected using echoplanar imaging with free induction decay for three blocks of 360 whole-brain volumes (echo time $[\mathrm{TE}]=25 \mathrm{msec}$, repetition time $[\mathrm{TR}]=2,000 \mathrm{msec}$, flip angle $\left.=70^{\circ}\right)$ with 33 axial slices $(64 \times 64$ grid, $3.44 \times 3.44 \times 3 \mathrm{~mm}$ voxels, interleaved order, 3-mm thickness, 1-mm spacing). A structural scan was collected using three-dimensional TurboFLASH imaging with nonselective excitation $(\mathrm{TE}=3.93 \mathrm{msec}, \mathrm{TR}=$ 2,300 msec, flip angle $\left.=12^{\circ}\right)$ with 160 sagittal slices $(512 \times 448$ grid, $0.5 \times 0.5 \times 1.0 \mathrm{~mm}$ voxels, $1-\mathrm{mm}$ thickness). Functional volumes were checked for transient spike artifacts, and the participants whose data sets contained unacceptable numbers of spike artifacts were excluded from further analysis (see the supplemental materials for details).

Preprocessing was done using SPM5 (Wellcome Trust Centre for Neuroimaging, 2005) with default parameters except where otherwise specified. The structural scan was skull-stripped using BET2 (Péchaud, Jenkinson, \& Smith, 2006). The functional images were slice-timing corrected using Fourier phase-shift interpolation with the first slice as reference and then motion corrected and resliced using least-squares 6-parameter rigid-body transformation. The structural scan was then coregistered with the functional scans using affine transformation and resliced. The images were then normalized and written to Montreal Neurological Institute (MNI) space with 12-parameter affine registration followed by a nonlinear deformation ( 25 iterations, nonlinear regularization $=10)$ with the structural scan as source image and SPM5's MNI Avg152 T1 at $2 \mathrm{~mm}^{3}$ with associated weighting mask as template. Finally, the normalized images were smoothed with an $8-\mathrm{mm}^{3}$ FWHM Gaussian kernel.

Intraparticipant analysis. The first-level analysis of the preprocessed fMRI data was performed using SPM5. A general linear model (GLM) was run for each participant with a canonical hemodynamic response function with no derivatives, a microtime resolution of 16 time bins per scan, a high-pass filter cutoff at $128 \mathrm{sec}$ using a residual forming matrix, autoregressive AR(1) to account for serial correlations, and restricted maximum likelihood (ReML) for model estimation. The model included a constant term, 6 motion regressors using the parameters of the motion correction performed during preprocessing, and 13 event-related regressors to model activation during the decision period and the outcome period.

The decision period for each trial was classified on whether the message for the block was the control (Control), positively framed (Positive), or negatively framed (Negative) message and whether the deck selected was good $(\mathrm{Good})$ or bad $(\mathrm{Bad})$. This provided six regressors (ControlGood, ControlBad, PositiveGood, PositiveBad, NegativeGood, and NegativeBad) plus a seventh regressor (NoResponse) for trials in which no response was made, regardless of the message. The decision-making events were aligned to the time of response.

The outcome period for each trial was classified on whether a good $(\mathrm{Good})$ or bad $(\mathrm{Bad})$ deck was selected and whether the actual outcome was a gain (Win), a draw (Draw), or a loss (Lose). Note that draws were only possible after good decisions because of the design of the decks. This provided five regressors (GoodWin, BadWin, GoodLose, BadLose, and GoodDraw) plus a sixth regressor (NoResponseOutcome) for trials in which no response was made. The outcome events were aligned to the time of presentation of the outcome.

Contrasts of interest were defined for changes in brain activity during the decision-making period. The risk effect was defined as the difference in brain activation associated with choosing a bad deck versus choosing a good deck. For comparison of activations with a framed message versus with the control message, the heightenedrisk effect was defined as the difference in the risk effect for a framed-message block compared with the control-message block. Finally, to determine whether the heightened-risk effect was driven by changes in brain activation associated with good or bad decisions, deck-specific contrasts compared activations between blocks for only good or bad decisions.

Group analysis. The second-level analyses used linear regression on the per-participant measures with ReML estimation 
in SPM5. Linear regressions were used because they show that brain activations are predictive of behavioral performance on an individual-by-individual basis. To identify regions whose activity related to choice behavior without informative messages, a correlation was calculated across participants in control-message blocks between decision quality and the risk effect. To identify regions whose activity related to changes in choice behavior due to the informative messages versus the control message, correlations were computed between message effectiveness and the heightened-risk effect. Finally, to identify regions showing a behaviorally relevant risk effect across all messages, correlations were computed between average decision quality across blocks and the average risk effect. The statistical threshold for significance was $p<.05$, with familywise error (FWE) correction using random field theory. The use of strong whole-brain correction for exploratory correlation analyses renders the recent criticism of correlation measures inapplicable (Lieberman, Berkman, \& Wager, 2009). Solely for visualization in figures, we used a threshold of $p<.0005$, uncorrected, with maximum color brightness indicating $p<.000005$, uncorrected, with the further constraint that only voxels contiguous with those passing the $p<.05$, FWE, threshold were shown. Anatomical labeling within the images was done using the Talairach Daemon (Lancaster et al., 2000) and anatomical automatic labeling (Tzourio-Mazoyer et al., 2002) for reference.

ROI analysis. Follow-up ROI analyses were performed using SPM5 and MarsBaR (Brett, Anton, Valabregue, \& Poline, 2002) within significant regions from the group analyses described above. Voxels that passed a whole-brain FWE-corrected threshold stand on their own as significant findings. The ROI analyses were used to illustrate and provide further details about the significant relationships found using the whole-brain voxel-by-voxel tests described above. When the follow-up contrasts are not orthogonal to the contrasts used to identify the ROI, the results are not independent, the effect sizes of these correlations are biased by the selection method, and the values should be considered only a descriptive summary (Lieberman et al., 2009).

Regions were defined as contiguous voxels that each passed the $p<.05$, FWE, threshold. Mean parameter estimates within ROIs are reported as percent magnetic resonance (MR) signal change calculated as the mean magnitude of the event regressor relative to the mean magnitude of the constant term regressor within the region. Follow-up analyses were calculated as the correlation between behavioral measures of decision quality or message effectiveness and neural measures of the risk effect or heightened-risk effect. A threshold of $p<.05$, uncorrected, was used in significance tests. Steiger's $Z$ was used when comparing two correlations that shared a common variable (Meng, Rosenthal, \& Rubin, 1992). The PearsonFilon statistic modified to use Fisher's $r$-to- $Z$ transformation (ZPF) was used when comparing two correlations that did not share a variable but used measures from the same set of participants (Raghunathan, Rosenthal, \& Rubin, 1996).

Mediation analysis. Two analyses were performed using the Mediation Toolbox (Wager et al., 2008) to test hypotheses about mediators of the relationship across participants between brain activity in an ROI and choice behavior with an informative message. In both analyses, the predictor was the risk effect in an ROI during an informative message block, and the outcome was the decision quality in that block. In the standard analysis, the potential mediator was the risk effect in a second ROI, hypothesized to mediate the relationship between the first ROI and choice. In the mediation effect parametric mapping (MEPM) analysis, a whole-brain search was performed, testing the risk effect in each voxel for mediation.

Significant mediation was defined as significance in three tests: indirect path $a$, relating the predictor and mediator; indirect path $b$, relating the mediator to outcome controlling for the predictor; and mediation effect $a b$, indicating that the predictor-outcome relationship is significantly reduced by the mediator (standard analysis, $p<.05$, using robust regression and bootstrapping with 1,000 samples; MEPM analysis, $p<.005$, using bootstrapping with 1,000 samples, and three contiguous voxels) (Baron \& Kenny, 1986; Wager et al., 2008).

\section{RESULTS}

\section{Behavioral Results}

Decision quality was significantly above chance with the control message $(M=0.26, S E=0.060)[t(26)=$ $4.27, p<.0005]$, indicating that even with the control message, the participants picked more good decks than bad decks. Relative to the control message, decision quality was significantly higher with both the positively framed message $(M=0.47, S E=0.078)[t(26)=2.82$, $p<.01]$ and the negatively framed message $(M=0.53$, $S E=0.076)[t(26)=3.63, p<.005]$, indicating that both framed messages were effective in improving choice behavior (see Figure 2A). Decision quality did not differ between the framed messages $[t(26)=-0.84, p=.41]$.

In order to evaluate whether the influence of the informative messages and the knowledge gained from experi-

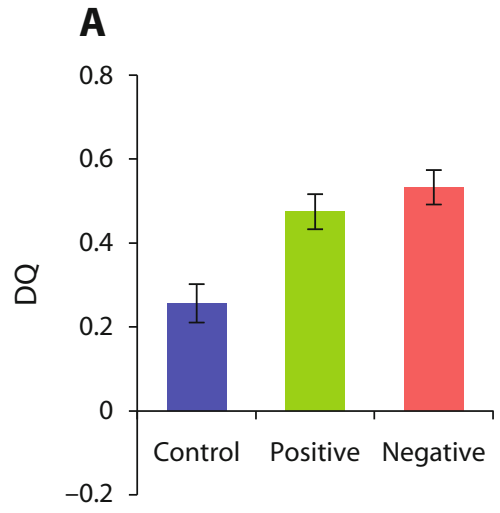

Message

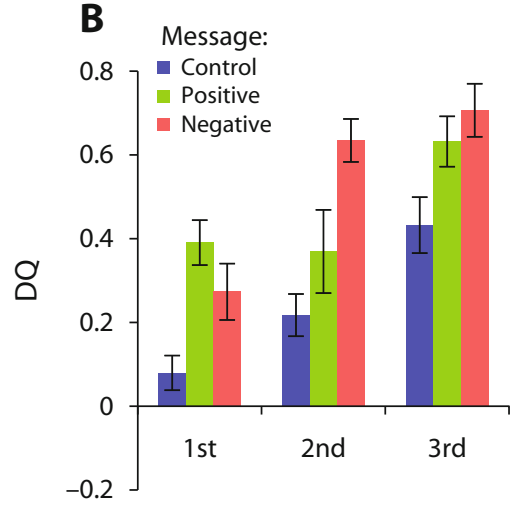

Block Position

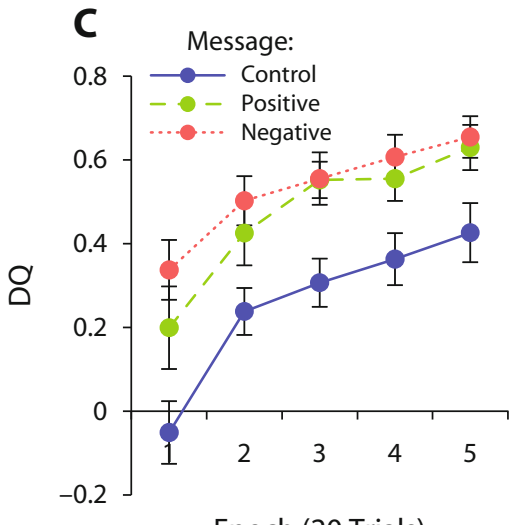

Epoch (20 Trials)

Figure 2. Decision quality (DQ). There were significant main effects on DQ of the message presented, the block position within the session, and the epoch within each block of trials. None of the interactions were significant. Error bars indicate within-subjects $S E M$ data (Loftus \& Masson, 1994) for panels A and C and between-subjects $S E M$ for panel B. (A) DQ as a function of message. (B) DQ as a function of message and block position within the session. (C) DQ as a function of message and epoch within the block of trials. 
ence interacted in their effects on decision quality, we ran a three-way within-subjects ANOVA with message, block position within session, and epoch within block as factors. Although all of the main effects were significant, none of the interactions were, suggesting that the effectiveness of the messages was additive with learning from experience within and across trial blocks (see Figures $2 \mathrm{~B}$ and $2 \mathrm{C}$ and Supplemental Table S1).

\section{fMRI Results}

We focus on between-subjects correlations of behavioral measures and neural contrasts. These relationships across participants provide a critical link between neural activity and behavior that is stronger than simple main effects analysis (see Supplemental Figure S1, Supplemental Table S2, and the supplemental text for reporting of main effects).

Positively framed message. For the positively framed informative message, there is a significant positive correlation between message effectiveness and the heightenedrisk effect in the right AI (Brodmann area [BA] 13, peak voxel: MNI coordinates 34, 24, 8) $[t(25)=6.65, p<.05$, FWE] (see Figure 3A). Summary ROI analysis illustrates that within this region, for the positively framed message
A

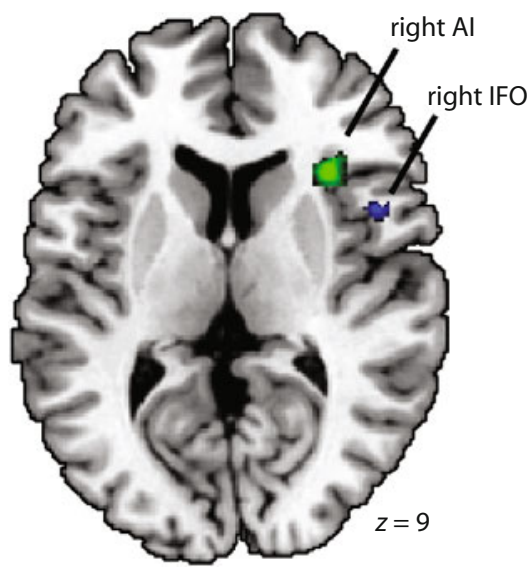

C

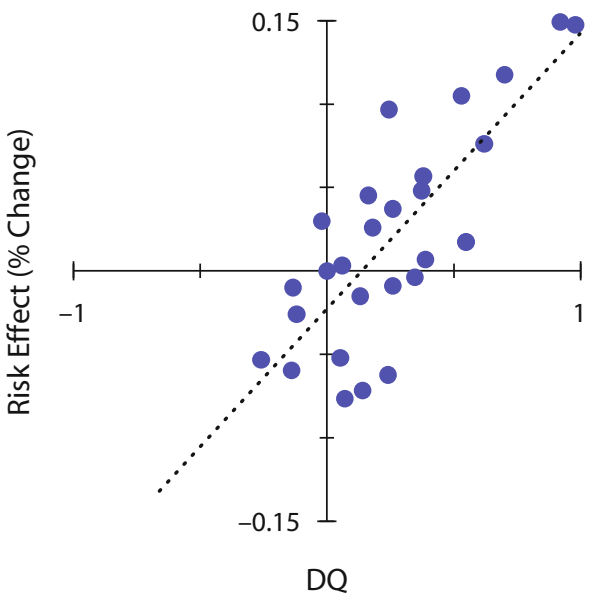

B

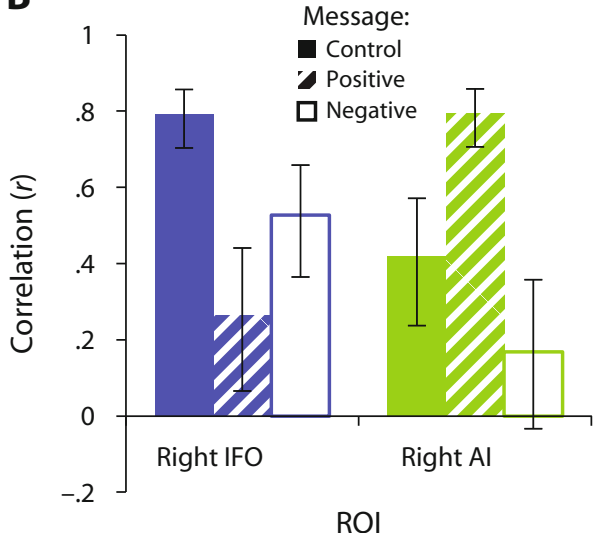

D

Right Al: Positive Message

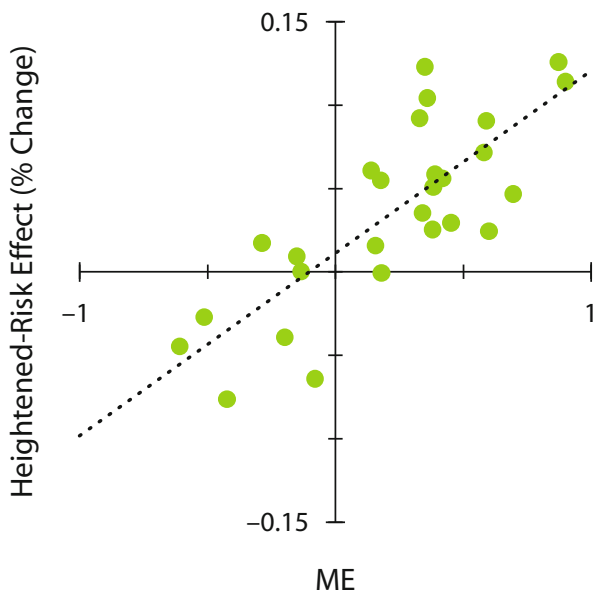

Figure 3. Correlations of behavioral and neural measures during control and positively framed message blocks. (A) A region of the right IFO (BA 44, peak voxel: MNI 48, 10, 10) in which, for the control message, there was a correlation between decision quality (DQ) and the risk effect, and a region of the right AI (BA 13, peak voxel: MNI 34, 24, 8) in which, for the positively framed message relative to the control message, there was a correlation between message effectiveness (ME) and the heightened-risk effect. Transverse section of the human brain (MNI $z=9$ ). The skull-stripped single-subject MNI CH2BET template was used as the background brain image in this and all subsequent figures. (B) Follow-up ROI analyses in the two regions described in panel $A$ for the correlation of $D Q$ and the risk effect with the control message (solid), the correlation of $\mathrm{ME}$ and the heightened-risk effect with the positively framed message (hatched), and the correlation of ME and the heightened-risk effect with the negatively framed message (outline). Note that the definition of these ROIs biases these comparisons in the direction found. Error bars indicate SEM. (C \& D) Plots of the control message correlation in the right IFO (C) and the positively framed message correlation in the right $\mathrm{AI}$ (D). Each point represents a participant. The dotted lines show the best-fit linear regressions. 
A

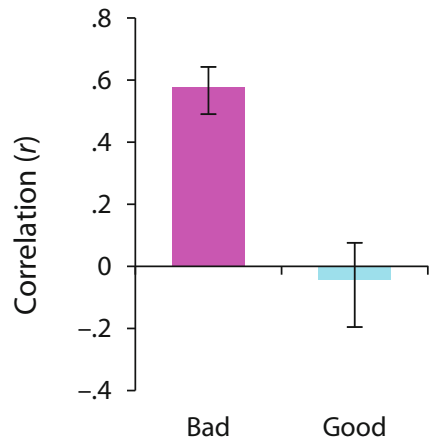

Deck Selected
B

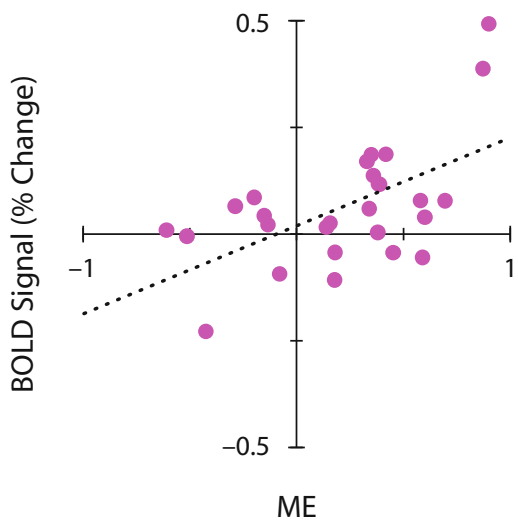

C

Good Deck

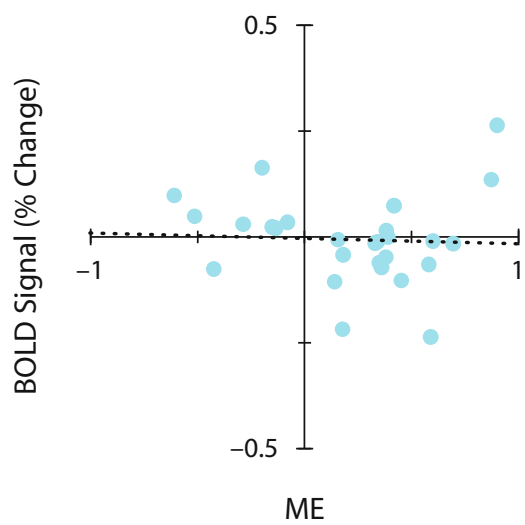

Figure 4. ROI analysis for the right AI region shown in Figure 3A identified by the correlation of message effectiveness (ME) and the heightened-risk effect for the positively framed message. (A) Correlation between ME and activation associated with selections from bad decks (darker/magenta) and good decks (lighter/cyan) for the positively framed message compared with the control message. Error bars indicate 1 SEM. (B \& C) Plots of the correlations shown in panel A for selections from bad decks (B) and good decks (C). Each point represents a participant. The dotted lines show the best-fit linear regressions.

compared with the control message, a greater increase in the risk effect is associated with a greater increase in decision quality $[r(25)=.79]$ (see Figure 3D). In other words, the right AI mediates the relationship between the influence of the positively framed message and the resulting improvement in decision quality.

Within the ROI in the right AI, we found that the correlation between positively framed message effectiveness and the heightened-risk effect was due to an increase in the BOLD activation for bad decisions $[r(25)=.58, p<$ $.005]$, with no change in activation for good decisions $[r(25)=-.04, p=.83]$ (see Figure 4). The difference between these correlations was significant (Steiger's $Z=$ $3.80, p<.0005)$. This suggests that the positively framed message improved decision quality by increasing the neural representation of appraised risk in right $\mathrm{AI}$ associated with selecting from the bad decks.

Negatively framed message. No brain areas showed a significant positive correlation between message effectiveness and the heightened-risk effect for the negatively framed message. This was the case even when a more relaxed statistical correction $(p<.05)$, false discovery rate, was used in place of the stringent FWE correction. Only when an uncorrected test was used was an active region identified in the left middle frontal gyrus (BA 6, peak voxel: MNI $-20,-2,62)[t(25)=4.49, p<.0001$, uncorrected] (see Supplemental Figure S2).

Control message. Considering only blocks with the control message, we found a significant positive correlation between decision quality and the risk effect in the right IFO (BA 44, peak voxel: MNI 48, 10, 10) $[t(25)=$ $7.18, p<.05$, FWE] (see Figure 3A). Within this area, summary ROI analysis illustrates that a greater risk effect is associated with higher decision quality $[r(25)=.79]$ (see Figure 3C). Since the control message does not provide information about specific decks, knowledge about the relative riskiness and long-term payoff of the decks came only from the results of past trials within the block, past blocks, and general prior experience.

We sought to further elucidate the roles of the right IFO and the right $\mathrm{AI}$ in risk appraisal based on past experience and due to framed informative messages (Figure 3B). In the right IFO, the correlation of the risk effect and decision quality with the control message was larger than the correlation of the heightened-risk effect and message effectiveness with the positively framed message ( $\mathrm{ZPF}=$ $3.07, p<.005)$. The converse was found in the right AI $(\mathrm{ZPF}=2.51, p<.05)$. Furthermore, the correlation of the heightened-risk effect and message effectiveness with the negatively framed message was marginally less than the control message correlation in right IFO $(\mathrm{ZPF}=1.70$, $p<.1)$ and significantly less than the positively framed message correlation in right $\mathrm{AI}(\mathrm{ZPF}=3.00, p<.005)$. These results suggest a degree of specialization, with the right IFO more involved in learning to anticipate adverse outcomes from experience and the right AI more involved in anticipating adverse outcomes on the basis of positively framed informative messages. However, caution is warranted for this conclusion because these comparisons were biased in the direction found by the nonindependent selection criteria for the ROIs.

Averaging across messages. Finally, we identified brain areas that may play an integrating role in decision making. We tested for regions showing a positive correlation between the risk effect and decision quality averaging across all three messages. Two brain regions met this criterion: one in the ACC and the dorsomedial prefrontal cortex (DMPFC; BA 6/32, peak voxel: MNI -6, 28, 38) $[t(25)=8.06, p<.05, \mathrm{FWE}]$ (see Figure 5A) and one in the left dorsolateral prefrontal cortex (DLPFC; BA 9, peak voxel: MNI $-44,12,40)[t(25)=6.91, p<.05$, FWE]. 
A

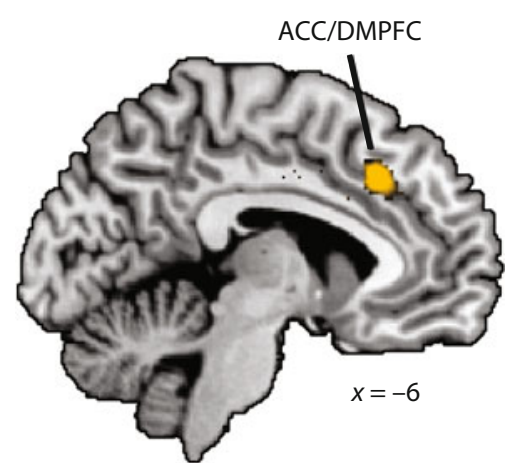

D

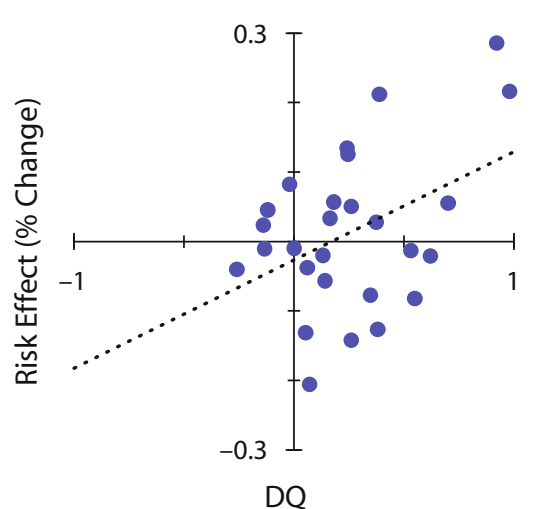

B

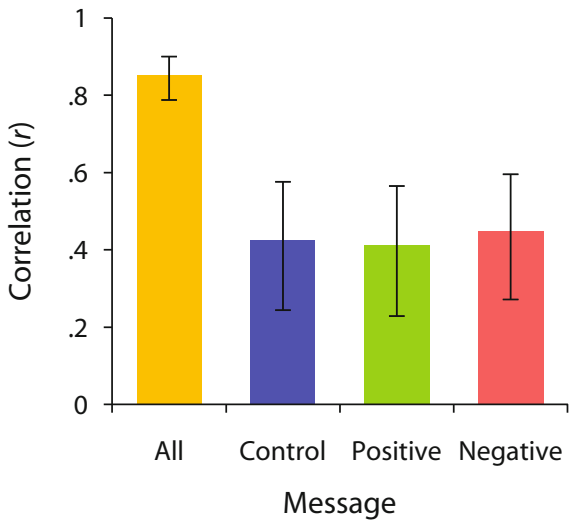

E

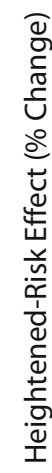

Positive Message

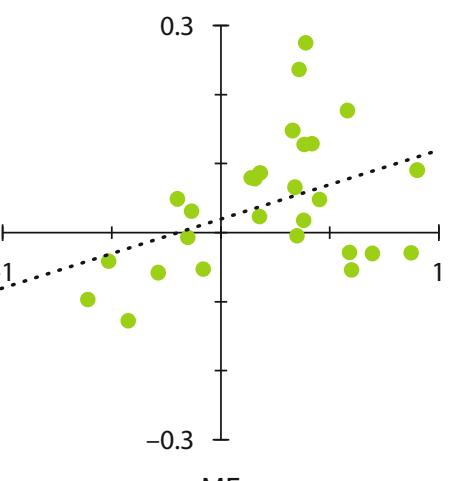

C

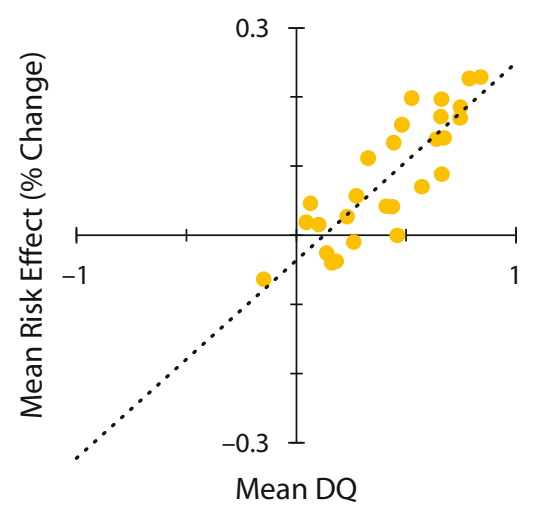

$\mathbf{F}$

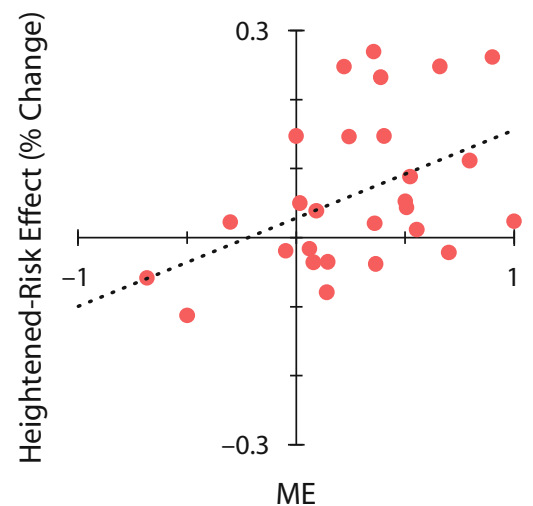

Figure 5. ACC and MPFC regions showing correlations of decision quality (DQ) and the risk effect averaging across all messages. (A) A region of the left ACC and the MPFC (BA 6/32, peak voxel: MNI -6, 28, 38) in which, averaging across messages, there was a correlation between DQ and the risk effect. Sagittal section of the human brain (MNI $x=-6$ ). (B) Within the region shown in panel A, confirmatory ROI analysis illustrates that DQ is highly correlated with the risk effect when averaging across all messages. In addition, when considering only the control message, DQ is correlated with the risk effect, and for both the positively framed and the negatively framed informative messages, message effectiveness (ME) was correlated with the heightened-risk effect. Error bars indicate $1 S E M$. (C) The correlation of DQ and the risk effect averaged across messages. (D) The correlation of DQ and the risk effect with the control message. ( $E \&$ F ) The correlation of $M E$ and the heightened-risk effect with both the positively framed $(E)$ and negatively framed (F) messages. For panels $\mathrm{C}-\mathrm{F}$, each point represents a participant, and the dotted lines show the best-fit linear regressions.

Follow-up ROI analysis in the ACC/DMPFC region illustrated that, averaging across all of the blocks, the greater the risk effect, the higher the decision quality $[r(25)=.85]$ (see Figures 5B and 5C). The correlation of risk effect and decision quality held when considering only the control message blocks $[r(25)=.42, p<.05]$ (see Figures 5B and 5D). In addition, the correlations of the heightened-risk effect and message effectiveness with both the positively framed message $[r(25)=.41, p<.05]$ and the negatively framed message $[r(25)=.45, p<.05]$ were significant and of similar magnitude within the ROI (see Figures 5B, 5E, and 5F). These results are consistent with the ACC aggregating risk appraisals from multiple sources of information, including experience and informative messages. Follow-up ROI analysis in the DLPFC region showed similar results (see the supplemental text for details).

Mediation analysis. We found relationships between the right $\mathrm{AI}$ and message effectiveness with the posi- tively framed message and between the ACC/DMPFC and DLPFC and decision quality with all messages. On the basis of these findings and previous anatomical, functional, and theoretical work (e.g., Barbas \& Pandya, 1989; Bechara \& Damasio, 2005; Craig, 2002; Kerns et al., 2004; MacDonald, Cohen, Stenger, \& Carter, 2000), we hypothesized that the ACC/DMPFC mediates the influence of the right $\mathrm{AI}$ on decision quality when the positively framed message is displayed. We tested this directly with standard mediation analyses on positively framed message blocks. As is shown in Figure 6A, the risk effect in the ACC/DMPFC ROI is a significant mediator of the relationship between the risk effect in the right AI ROI and decision quality $(p<.05$ for path $a$, path $b$, and mediation effect $a b$, using robust regression and bootstrapping with 1,000 samples). For comparison, we did the same analysis with the risk effect in the left DLPFC ROI as mediator, but this was not significant ( $p>.05$ for mediation effect $a b$ ). 


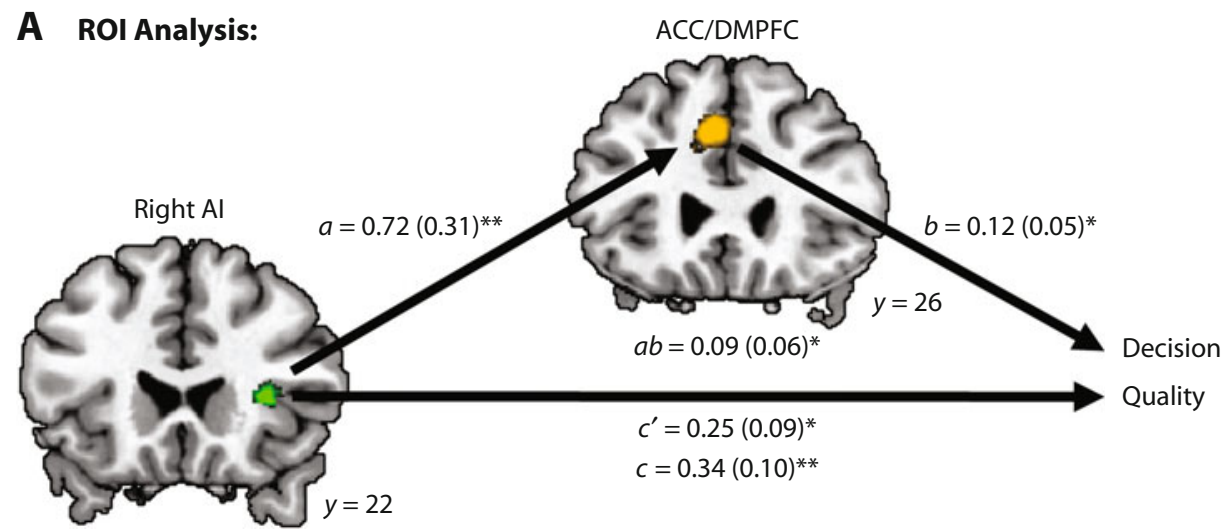

\section{B Whole-Brain Search:}

?

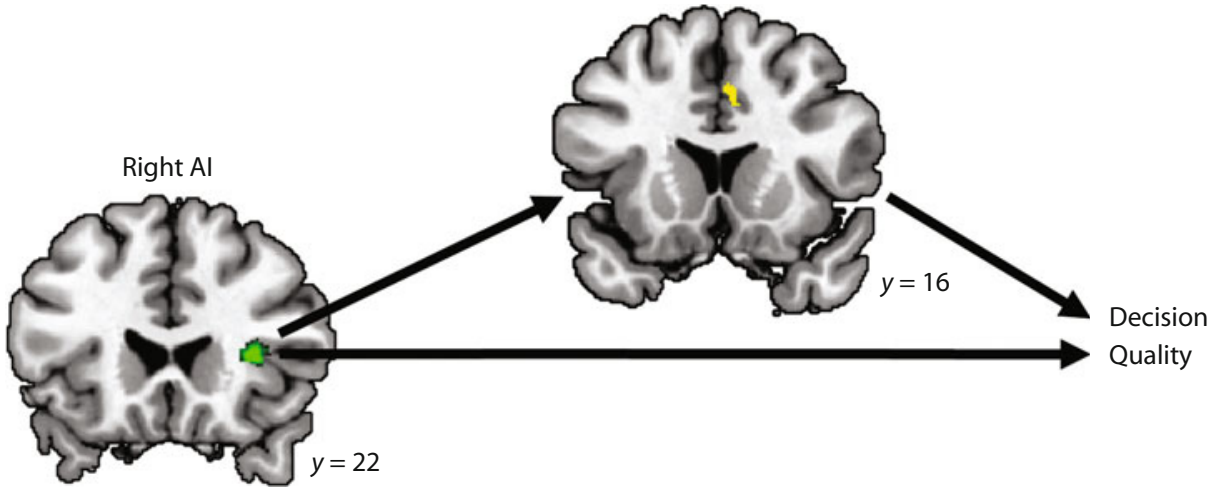

C

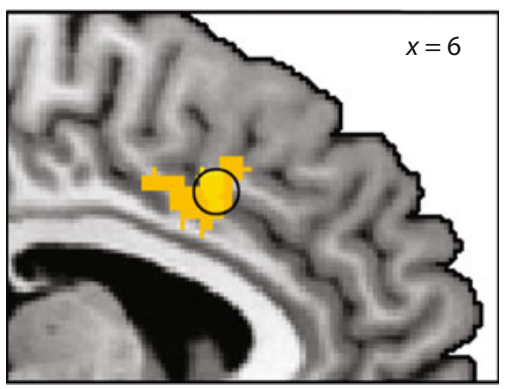

D

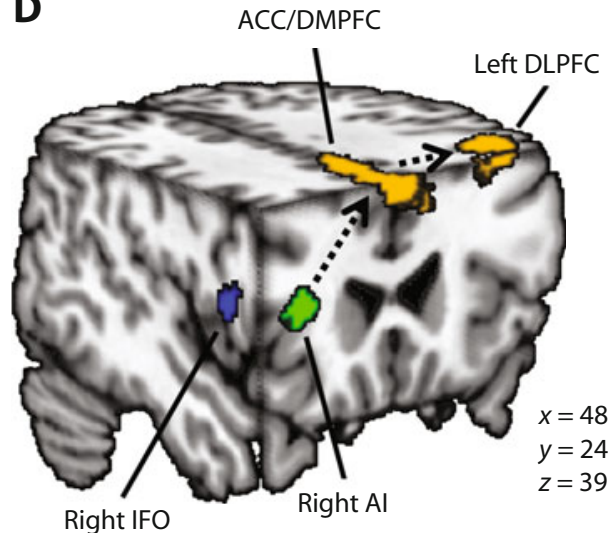

Figure 6. Analyses showing that, for the positively framed message, the ACC/MPFC mediates the relationship between the right $\mathrm{AI}$ and decision quality. (A) The ACC/MPFC ROI significantly mediates the relationship between the right $\mathrm{AI}$ ROI and decision quality. (B) This region (BA 32, peak voxel: MNI 6, 14, 40) is significant in a whole-brain voxel-by-voxel search for mediators of the relationship between the right AI ROI and decision quality (see Supplemental Table S3 for a list of all significant regions). (C) Close-up view showing overlap (circled) of the ACC/MPFC ROI and the region identified in the whole-brain mediation analysis. (D) Summary of regions identified in this study on an interior view of the human brain: right IFO identified from control message blocks, right $\mathrm{AI}$ identified from positively framed message blocks, ACC/MPFC and left DLPFC identified by averaging across all message blocks. Dotted lines indicate the proposed path for the influence of positively framed messages, supported by functional mediation analysis. ${ }^{*} p<.05$. ${ }^{* *} p<.01$. 
However, we did find that the risk effect in the ACC/ DMPFC ROI is a significant mediator of the relationship between the risk effect in the right AI ROI and the risk effect in the left DLPFC ROI ( path $a, M=0.72, S E=0.28$, $p<.01$; path $b, M=0.93, S E=0.21, p<.01$; mediation effect $a b, M=0.67, S E=0.31, p<.01$, using robust regression and bootstrapping with 1,000 samples).

As a follow-up, we performed a whole-brain MEPM analysis searching for voxels that, in positively framed message blocks, significantly mediated the relationship between the risk effect in the right AI ROI and decision quality. A number of brain regions were identified, including the ACC, the posterior cingulate, and the superior parietal lobule ( $p<.005$ for path $a$, path $b$, and mediation effect $a b$, three contiguous voxels; see Supplemental Table S3 for a full listing of regions). The region with the most significant mediation effect was in the ACC/DMPFC (BA 32, peak voxel: MNI 6, 14, 40) and overlaps with the ACC/DMPFC ROI previously identified (see Figures 6B and $6 \mathrm{C}$ ). No identified regions overlapped with the left DLPFC ROI.

\section{Excluding Alternative Interpretations}

We have ruled out a number of alternative explanations for the risk effects and heightened-risk effects. We list them here, with details in the supplemental text. (1) There is no "prominent deck B" phenomenon (Lin et al., 2007) hiding a preference for the net-loss/rare-loss deck, so we are justified in grouping the good decks and bad decks together to increase statistical power and simplify the analysis. (2) Additional GLMs demonstrate that the unequal distribution of selections from good and bad decks throughout each block of trials does not account for the risk effect. (3) The results cannot be explained by differences in response time among conditions or participants, because response time is not correlated with risk effects, heightened-risk effects, decision quality, or message effectiveness. (4) ANCOVAs exclude the possibility that differences in the order of message presentation explain the correlations between brain activity and behavior. (5) A rarity- or novelty-based explanation cannot account for the correlation of message effectiveness with the heightened-risk effect. By excluding these potential alternative explanations, risk appraisal remains the most reasonable explanation for our findings of differences in activation for choices from bad and good card decks.

\section{DISCUSSION}

We used event-related fMRI to investigate the neural basis of how positively and negatively framed informative messages influence choice behavior in the IGT, a task involving realistic decision making under uncertainty (Bechara et al., 1994).

\section{Effectiveness of Messages in Altering Choice Behavior}

We first established that the informative messages had the desired positive effect on choice behavior. Our behavioral measures were decision quality, indicating the pro- pensity to make good versus bad decisions, and message effectiveness, indicating the increase in decision quality when presented with the positively or negatively framed informative message relative to the control message. The participants' decision quality was better with both the positively and negatively framed informative messages than with the control message, indicating that both framed messages were effective and that the participants paid attention to them and incorporated them into the decisionmaking process.

We then addressed how message effectiveness interacted with learning from experience occurring within and between trial blocks. We found that the message effects did not interact with the effects of time within block or of block order, indicating that the informative messages and learning from past trials had independent additive effects on choice behavior. This result suggests that the informative messages did not preempt the decision-making process by causing the participants to cede to experimenter demand but, rather, provided an additional source of information that they incorporated into decision making.

Allowing the participants to learn the riskiness of each deck by experience instead of by being explicitly told the payoff distributions of each deck was desirable, because the participants were less likely to ignore the messages when choosing a deck. Nonetheless, the trade-off of allowing learning as opposed to using explicit prior description of the deck payoff probabilities is that the participants' appraisal of deck risk is colored by their individual differences in reward and punishment sensitivity during learning. In principle, this potentially conflates punishment sensitivity in learning with risk aversion in decision making, but in practice, the two effects may be two sides of the same coin. In any case, this is not a problem for the present study, because the range of preferences for the good versus bad decks simply provides useful variance that we exploit to identify the neural correlates of such individual differences specifically during the decision phase, regardless of the origin of the differences.

\section{Heightened-Risk Appraisals Correlated With Positively Framed Message Effectiveness}

Since the informative messages influenced choice behavior, we sought to identify brain regions associated with these effects. A wide range of recent work suggests a role for risk appraisal in decision making and has begun to uncover its neural basis (Behrens et al., 2007; Brown \& Braver, 2007; Fukui et al., 2005; Paulus \& Frank, 2006; Preuschoff et al., 2008). We looked for activity representing the appraised risk of the selected option. Our neural measure of risk appraisal was the risk effect, defined as greater activation when selecting from bad decks rather than good decks. The heightened-risk effect was defined as an increase in the risk effect when presented with an informative message rather than the control message.

As was predicted, we identified a region in the right AI in which message effectiveness was correlated with the heightened-risk effect for the positively framed message. In other words, the greater the enhancement of the risk effect in the right AI, the more effectively the positively 
framed message improved choice behavior. This suggests that the AI mediated the influence of the positively framed informative message on choice behavior. Indeed, this analysis equates to a within-subjects mediation analysis with message as the predictor, decision quality as the outcome, and the risk effect in the right AI as the mediator (Judd, Kenny, \& McClelland, 2001; see the supplemental text for details).

The increased risk effect in the right $\mathrm{AI}$ associated with higher decision quality could be due to various changes in activity during good and bad decisions. For example, activation could increase during bad decisions, it could decrease during good decisions, it could do both, or it could even decrease during bad decisions while decreasing even more during good decisions. We found that improvements in decision quality due to the positively framed message were correlated with increases in AI activity when the participants selected from the bad decks but were not associated with a change in activity when the participants selected from the good decks. Thus, even though the positively framed message was focused on the advantages of the good decks, this information was apparently used to appraise the bad decks as more risky by comparison.

This result is consistent with prospect theory, which predicts that the alteration of a reference frame used for risk appraisal will lead to changes in that appraisal (Rothman \& Salovey, 1997). It also provides neural evidence consistent with an idea in the field of health behavior change: In circumstances in which people believe that they have a positive situation to maintain, an effective way to help people appreciate the negative consequences of risky behavior is to emphasize the positive consequences of alternative behavior (Rothman \& Salovey, 1997).

\section{Specialization Within the AI}

We identified a nearby region in the right IFO where decision quality is positively correlated with the risk effect in trial blocks using only the control message. This result suggests that the right IFO plays a role in the evaluation of risk on the basis of the history of reward and punishment and other past knowledge of the task and context. The AI and the IFO are often considered a single module involved in the representation of subjective feeling states, because they jointly receive the same afferent connections from subcortical areas and jointly form part of the gustatory cortex (Craig, 2002; Jabbi, Swart, \& Keysers, 2007). However, our findings suggest a partial differentiation of the right IFO and the right AI that warrants further investigation: between internal experience-based risk appraisal in the right IFO and external message-influenced risk appraisal in the right AI. This is also supported by our finding of additive effects of the influence of the framed informative messages and learning from experience on decision quality. Further work will be required to determine whether this differentiation of the AI and the IFO will generalize to other decision-making contexts and messages or is particular to the task and materials used here.

\section{Lack of Correlations With \\ Negatively Framed Message Effectiveness}

For the negatively framed message, contrary to our predictions, we did not identify any brain areas in which message effectiveness was significantly correlated with the heightened-risk effect. We did identify a region in the left middle frontal gyrus, but only when we removed the appropriate correction for multiple comparisons. Behaviorally, the negatively framed message was as effective as the positively framed message in improving choice behavior. However, past work suggests that there could be a crossover interaction between message frame and an individual-difference measure such as attitude toward risk, which could lead to the lack of a main effect of message frame despite systematic underlying differences in individuals (Mann, Sherman, \& Updegraff, 2004). This could explain a difference between positive and negative frames, but it does not address the lack of significant findings.

One possibility is that there may have been high variability across participants in the brain regions involved in the improvement in decision quality with the negatively framed message. Individual differences, including gender (Toll et al., 2008), need for cognition (Steward, Schneider, Pizarro, \& Salovey, 2003), and attitude toward risk (Mann et al., 2004) can interact with the effectiveness of particular message frames. Thus, a hypothesis to pursue in the future is that the brain regions mediating the effectiveness of negatively framed messages differ as a function of these individual-difference measures.

Note that ROI analysis in the ACC/DMPFC region identified by averaging across all messages revealed a significant correlation between message effectiveness and the heightened-risk effect for the negatively framed message. Further experimentation is necessary to determine why we failed to find voxels showing this relationship in the whole-brain search.

\section{Risk Appraisals Correlated With Decision Quality Across Messages}

Averaging across all of the messages, we identified regions of the ACC/DMPFC and DLPFC in which decision quality correlated with the risk effect. Within the ACC/ DMPFC region, we found similarly significant correlations between decision quality and the risk effect with the control message and between message effectiveness and the heightened-risk effect for both of the framed messages. This suggests a role for the ACC/DMPFC in integrating risk appraisals due to informative messages with those from other sources of information. This is consistent with a developing account of this region as central to actionrelevant evaluation of risk and its avoidance (Brown \& Braver, 2008; Magno et al., 2006; Paulus \& Frank, 2006).

The DLPFC, for which we also found a correlation between decision quality and the neural risk effect across messages, is involved in the implementation of top-down cognitive control over behavior (Miller \& Cohen, 2001). This has typically been conceived in terms of biasing signals sent to other regions in which action selection occurs. A number of studies support the idea that although the 
ACC/DMPFC is more directly involved in the evaluation of risk, the DLPFC is more directly involved in the implementation of top-down cognitive control as a result of that evaluation (Barbas \& Pandya, 1989; Kerns et al., 2004; MacDonald et al., 2000).

\section{A Network for the Influence of Informative Messages on Decision Making}

As a final step, we used mediation analysis (Baron \& Kenny, 1986; Wager et al., 2008) to confirm our hypothesis that the relationship between the heightened-risk effect in the right AI and positively framed message effectiveness is mediated by the ACC/DMPFC. In contrast, we did not find such a direct role for the DLPFC; rather, the relationship between the right $\mathrm{AI}$ and the left DLPFC is also mediated by the ACC/DMPFC. This result suggests that the influence of informative messages on decision making is operating through a network with the AI, as part of a limbic sensory cortex, representing abstract and subjective feeling states, leading to the ACC, as a limbic motor cortex, actively guiding and motivating action (Barbas \& Pandya, 1989; Craig, 2002), with the DLPFC further downstream (Barbas \& Pandya, 1989; Kerns et al., 2004; MacDonald et al., 2000). Messages modulate risk appraisals that are instantiated as "as if" feeling states in the AI and then integrated for action selection in the ACC (Bechara \& Damasio, 2005; Dunn et al., 2006).

\section{Limitations and Future Directions}

In order to maximize our detection of changes in risk appraisal due to informative messages no matter the particular representation underlying them, we did not discriminate among the many proposed definitions of risk (for examples, see Yates, 1992). Note that a comparison between the two bad decks would control for expected value while manipulating both variance and adverse outcome probability, but we did not explore messages that discriminated between the two bad decks. However, the neural representation of risk is an important topic in its own right, and a full understanding of how messages interact with risk processing will depend on it. Furthermore, we cannot resolve whether the identified areas show risk appraisals that precede and drive choice behavior or merely follow and reflect it (Dunn et al., 2006). One fruitful approach may be hidden process models that determine when risk appraisal occurs relative to choice response with high temporal resolution (Hutchinson, Niculescu, Keller, Rustandi, \& Mitchell, 2009).

We did not find behavioral evidence of an interaction between message effects and learning effects in this study, and we limited our analysis to the period of choice selection. However, in general, informative messages may also affect the experience of outcomes and the learning that results, necessitating their inclusion in an overall understanding of persuasive messages. As a form of social communication, persuasive messages can be considered in the context of Lasswell's (1948) classic formulation, "Who says what in what channel to whom with what effect?" (p. 37). We manipulated the informational value and fram- ing of content, with choice behavior as our dependent variable. Future work will be needed to investigate manipulations of source, channel, receiver, and further dimensions of content, and to measure attitude change, which need not be correlated with behavioral preference (e.g., McClure et al., 2004).

In summary, we have presented evidence that the AI, the ACC/DMPFC, and the DLPFC form part of a network of brain areas underlying improvements in choice behavior due to informative messages by way of heightened risk appraisals (see Figure 6D). This work provides an initial neuroscientific basis for understanding the effect of persuasive messages on decision making under uncertainty. We hope that in the future, this work will lead to the development of more effective public health campaigns against risky behavior and to a better understanding of message effects on decision making more generally.

\section{AUTHOR NOTE}

This research was supported in part by National Institutes of Health Grant R03 DA023462-01 (J.W.B.), Air Force Office of Scientific Research Grant FA9550-07-1-0454 (J.W.B.), a 2005 NARSAD Young Investigator Award and the Sydney R. Baer, Jr. Foundation (J.W.B.), National Institutes of Health Grant T32 MH019879-15 (A.K.), and the Indiana METACyt Initiative of Indiana University, funded in part through a major grant from the Lilly Endowment, Inc. We thank Srikanth Padmala, Luiz Pessoa, Robert J. Rydell, Steven J. Sherman, and our anonymous reviewers for helpful comments and Elizabeth Dinh for help with data collection. Correspondence concerning this article should be addressed to J. W. Brown, Department of Psychological and Brain Sciences, Indiana University, 1101 East 10th Street, Bloomington, IN 47405 (e-mail: jwmbrown@indiana.edu).

\section{REFERENCES}

Barbas, H., \& PANDYA, D. N. (1989). Architecture and intrinsic connections of the prefrontal cortex in the rhesus monkey. Journal of Comparative Neurology, 286, 353-375. doi:10.1002/cne.902860306

BARON, R. M., \& KENNY, D. A. (1986). The moderator-mediator variable distinction in social psychological research: Conceptual, strategic, and statistical considerations. Journal of Personality \& Social Psychology, 51, 1173-1182. doi:10.1037/0022-3514.51.6.1173

Bechara, A., \& Damasio, A. R. (2005). The somatic marker hypothesis: A neural theory of economic decision. Games \& Economic Behavior, 52, 336-372. doi:10.1016/j.geb.2004.06.010

Bechara, A., Damasio, A. R., Damasio, H., \& Anderson, S. W. (1994). Insensitivity to future consequences following damage to human prefrontal cortex. Cognition, 50, 7-15. doi:10.1016/0010 $-0277(94) 90018-3$

Bechara, A., Damasio, H., Tranel, D., \& Damasio, A. R. (1997). Deciding advantageously before knowing the advantageous strategy. Science, 275, 1293-1295. doi:10.1126/science.275.5304.1293

Bechara, A., Dolan, S., Denburg, N., Hindes, A., Anderson, S. W., \& Nathan, P. E. (2001). Decision-making deficits, linked to a dysfunctional ventromedial prefrontal cortex, revealed in alcohol and stimulant abusers. Neuropsychologia, 39, 376-389. doi:10.1016/ S0028-3932(00)00136-6

Behrens, T. E. J., Woolrich, M. W., Walton, M. E., \& Rushworth, M. F. S. (2007). Learning the value of information in an uncertain world. Nature Neuroscience, 10, 1214-1221. doi:10.1038/nn1954

Brett, M., Anton, J.-L., Valabregue, R., \& Poline, J.-B. (2002). Marseille Boîte à Région d'Intérêt (MarsBaR). Retrieved September 1, 2007 from http://marsbar.sourceforge.net/.

Brown, J. W., \& Braver, T. S. (2005). Learned predictions of error likelihood in the anterior cingulate cortex. Science, 307, 1118-1121. doi:10.1126/science.1105783

Brown, J. W., \& Braver, T. S. (2007). Risk prediction and aversion by 
anterior cingulate cortex. Cognitive, Affective, \& Behavioral Neuroscience, 7, 266-277. doi:10.3758/CABN.7.4.266

Brown, J. W., \& Braver, T. S. (2008). A computational model of risk, conflict, and individual difference effects in the anterior cingulate cortex. Brain Research, 1202, 99-108. doi:10.1016/ j.brainres.2007.06.080

Chiu, Y.-C., \& LiN, C.-H. (2007). Is deck C an advantageous deck in the Iowa Gambling Task? Behavioral \& Brain Functions, 3, 37. doi:10.1186/1744-9081-3-37

Chiu, Y.-C., Lin, C.-H., Huang, J.-T., Lin, S., Lee, P.-L., \& Hsieh, J.-C. (2008). Immediate gain is long-term loss: Are there foresighted decision makers in the Iowa Gambling Task? Behavioral \& Brain Functions, 4, 13. doi:10.1186/1744-9081-4-13

Craig, A. D. (2002). How do you feel? Interoception: The sense of the physiological condition of the body. Nature Reviews Neuroscience, $\mathbf{3}$, 655-666. doi:10.1038/nrn894

Dale, A. M. (1999). Optimal experimental design for event-related fMRI. Human Brain Mapping, 8, 109-114. doi:10.1002/(SICI)1097 -0193(1999)8:2/3<109::AID-HBM7>3.0.CO;2-W

De Martino, B., Kumaran, D., Seymour, B., \& Dolan, R. J. (2006). Frames, biases, and rational decision-making in the human brain. $\mathrm{Sci}$ ence, 313, 684-687. doi:10.1126/science. 1128356

Dunn, B. D., Dalgleish, T., \& Lawrence, A. D. (2006). The somatic marker hypothesis: A critical evaluation. Neuroscience \& Biobehavioral Reviews, 30, 239-271. doi:10.1016/j.neubiorev.2005.07.001

Fishbein, D. H., Eldreth, D. L., Hyde, C., Matochik, J. A., LonDon, E. D., Contoreggi, C., ET AL. (2005). Risky decision making and the anterior cingulate cortex in abstinent drug abusers and nonusers. Cognitive Brain Research, 23, 119-136. doi:10.1016/ j.cogbrainres.2004.12.010

Fukui, H., Murai, T., Fukuyama, H., Hayashi, T., \& Hanakawa, T. (2005). Functional activity related to risk anticipation during performance of the Iowa Gambling Task. NeuroImage, 24, 253-259. doi:10.1016/j.neuroimage.2004.08.028

Gehring, W. J., Himle, J., \& Nisenson, L. G. (2000). Action-monitoring dysfunction in obsessive-compulsive disorder. Psychological Science, 11, 1-6. doi:10.1111/1467-9280.00206

Hutchinson, R. A., Niculescu, R. S., Keller, T. A., Rustandi, I., \& MitCHELl, T. M. (2009). Modeling fMRI data generated by overlapping cognitive processes with unknown onsets using Hidden Process Models. NeuroImage, 46, 87-104. doi:10.1016/j.neuroimage.2009 .01 .025

JabBI, M., Swart, M., \& Keysers, C. (2007). Empathy for positive and negative emotions in the gustatory cortex. NeuroImage, 34, 1744 1753. doi:10.1016/j.neuroimage.2006.10.032

Judd, C. M., Kenny, D. A., \& McClelland, G. H. (2001). Estimating and testing mediation and moderation in within-subject designs. Psychological Methods, 6, 115-134. doi:10.1037/1082-989X.6.2.115

Kato, J., Ide, H., Kabashima, I., Kadota, H., Takano, K., \& KanSAKU, K. (2009). Neural correlates of attitude change following positive and negative advertisements. Frontiers in Behavioral Neuroscience, 3, 6. doi:10.3389/neuro.08.006.2009

Kerns, J. G., Cohen, J. D., MacDonald, A. W., III, Cho, R. Y., Stenger, V. A., \& Carter, C. S. (2004). Anterior cingulate conflict monitoring and adjustments in control. Science, 303, 1023-1026. doi:10.1126/science. 1089910

Klucharev, V., Smidts, A., \& Fernández, G. (2008). Brain mechanisms of persuasion: How "expert power" modulates memory and attitudes. Social Cognitive Affective Neuroscience, 3, 353-366. doi:10.1093/scan/nsn022

KNIGHT, F. H. (1921). Risk, uncertainty, and profit. Boston: Houghton Mifflin.

Kuhnen, C. M., \& Knutson, B. (2005). The neural basis of financial risk taking. Neuron, 47, 763-770. doi:10.1016/j.neuron.2005.08.008

Lancaster, J. L., Woldorff, M. G., Parsons, L. M., Liotti, M., Freitas, C. S., Rainey, L., ET AL. (2000). Automated Talairach atlas labels for functional brain mapping. Human Brain Mapping, 10, 120 131. doi:10.1002/1097-0193(200007)10:3<120::AID-HBM30>3.0 . CO; $2-8$

LASSWELL, H. D. (1948). The structure and function of communication in society. In L. Bryson (Ed.), The communication of ideas, a series of addresses. New York: Institute for Religious and Social Studies.

Lawrence, N. S., Jollant, F., O’Daly, O., Zelaya, F., \& Phillips,
M. L. (2009). Distinct roles of prefrontal cortical subregions in the Iowa Gambling Task. Cerebral Cortex, 19, 1134-1143. doi:10.1093/ cercor/bhn 154

Lieberman, M. D., Berkman, E. T., \& Wager, T. D. (2009). Correlations in social neuroscience aren't voodoo: Commentary on Vul et al. (2009). Perspectives on Psychological Science, 4, 299-307. doi:10.1111/j.1745-6924.2009.01128.x

Lin, C.-H., Chiu, Y.-C., Cheng, C.-M., \& Hsieh, J.-C. (2008). Brain maps of Iowa gambling task. BMC Neuroscience, 9, 72. doi:10.1186/1471-2202-9-72

Lin, C.-H., Chiu, Y.-C., Lee, P.-L., \& Hsieh, J.-C. (2007). Is deck B a disadvantageous deck in the Iowa Gambling Task? Behavioral \& Brain Functions, 3, 16. doi:10.1186/1744-9081-3-16

Loewenstein, G. F., Weber, E. U., Hsee, C. K., \& Welch, N. (2001). Risk as feelings. Psychological Bulletin, 127, 267-286. doi:10.1037/0033-2909.127.2.267

LofTus, G. R., \& Masson, M. E. J. (1994). Using confidence intervals in within-subject designs. Psychonomic Bulletin \& Review, 1, 476490.

MacDonald, A. W., III, Cohen, J. D., Stenger, V. A., \& Carter, C. S. (2000). Dissociating the role of the dorsolateral prefrontal and anterior cingulate cortex in cognitive control. Science, 288, 1835-1838. doi:10.1126/science.288.5472.1835

Magno, E., Foxe, J. J., Molholm, S., Robertson, I. H., \& Garavan, H. (2006). The anterior cingulate and error avoidance. Journal of Neuroscience, 26, 4769-4773. doi:10.1523/JNEUROSCI.0369-06.2006

Mann, T., Sherman, D., \& UpdegrafF, J. (2004). Dispositional motivations and message framing: A test of the congruency hypothesis in college students. Health Psychology, 23, 330-334. doi:10.1037/0278 $-6133.23 .3 .330$

McClure, S. M., Li, J., Tomlin, D., Cypert, K. S., Montague, L. M., \& Montague, P. R. (2004). Neural correlates of behavioral preference for culturally familiar drinks. Neuron, 44, 379-387. doi:10.1016/ j.neuron.2004.09.019

Meng, X.-L., Rosenthal, R., \& Rubin, D. B. (1992). Comparing correlated correlation coefficients. Psychological Bulletin, 111, 172-175. doi:10.1037/0033-2909.111.1.172

Miller, E. K., \& Cohen, J. D. (2001). An integrative theory of prefrontal cortex function. Annual Review of Neuroscience, 24, 167-202. doi:10.1146/annurev.neuro.24.1.167

Northoff, G., Grimm, S., Boeker, H., Schmidt, C., Bermpohl, F., Heinzel, A., ET AL. (2006). Affective judgment and beneficial decision making: Ventromedial prefrontal activity correlates with performance in the Iowa Gambling Task. Human Brain Mapping, 27, 572-587. doi:10.1002/hbm.20202

O'Keefe, D. J., \& Jensen, J. D. (2007). The relative persuasiveness of gain-framed loss-framed messages for encouraging disease prevention behaviors: A meta-analytic review. Journal of Health Communication, 12, 623-644. doi:10.1080/10810730701615198

Paulus, M. P., \& Frank, L. R. (2006). Anterior cingulate activity modulates nonlinear decision weight function of uncertain prospects. NeuroImage, 30, 668-677. doi:10.1016/j.neuroimage.2005.09.061

Paulus, M. P., Rogalsky, C., Simmons, A., Feinstein, J. S., \& Stein, M. B. (2003). Increased activation in the right insula during risktaking decision making is related to harm avoidance and neuroticism. NeuroImage, 19, 1439-1448. doi:10.1016/S1053-8119(03)00251-9

Paulus, M. P., Tapert, S. F., \& Schuckit, M. A. (2005). Neural activation patterns of methamphetamine-dependent subjects during decision making predict relapse. Archives of General Psychiatry, 62, 761-768. doi:10.1001/archpsyc.62.7.761

Péchaud, M., Jenkinson, M., \& Smith, S. (2006). Brain Extraction Tool (BET) [Software]. Oxford: Oxford University Centre for Functional MRI of the Brain.

Preuschoff, K., Bossaerts, P., \& Quartz, S. R. (2006). Neural differentiation of expected reward and risk in human subcortical structures. Neuron, 51, 381-390. doi:10.1016/j.neuron.2006.06.024

Preuschoff, K., Quartz, S. R., \& Bossaerts, P. (2008). Human insula activation reflects risk prediction errors as well as risk. Journal of Neuroscience, 28, 2745-2752. doi:10.1523/JNEUROSCI.4286-07.2008

Raghunathan, T. E., Rosenthal, R., \& Rubin, D. B. (1996). Comparing correlated but nonoverlapping correlations. Psychological Methods, 1, 178-183. doi:10.1037/1082-989X.1.2.178

Rothman, A. J., \& SAlOVEY, P. (1997). Shaping perceptions to motivate 
healthy behavior: The role of message framing. Psychological Bulletin, 121, 3-19. doi:10.1037/0033-2909.121.1.3

SAATY, T. L. (1987). Risk - Its priority and probability: The analytic hierarchy process. Risk Analysis, 7, 159-172. doi:10.1111/j.1539-6924 .1987.tb00980.x

Steward, W. T., Schneider, T. R., Pizarro, J., \& Salovey, P. (2003). Need for cognition moderates responses to framed smoking-cessation messages. Journal of Applied Social Psychology, 33, 2439-2464. doi:10.1111/j.1559-1816.2003.tb02775.x

Suhr, J. A., \& Tsanadis, J. (2007). Affect and personality correlates of the Iowa Gambling Task. Personality \& Individual Differences, 43, 27-36. doi:10.1016/j.paid.2006.11.004

Toll, B. A., Salovey, P., O’Malley, S. S., Mazure, C. M., LatiMER, A., \& MCKEE, S. A. (2008). Message framing for smoking cessation: The interaction of risk perceptions and gender. Nicotine \& Tobacco Research, 10, 195-200. doi:10.1080/14622200701767803

Tversky, A., \& Kahneman, D. (1981). The framing of decisions and the psychology of choice. Science, 211, 453-458. doi:10.1126/ science. 7455683

Tzourio-Mazoyer, N., Landeau, B., Papathanassiou, D., Crivello, F., Etard, O., Delcroix, N., ET Al. (2002). Automated anatomical labeling of activations in SPM using a macroscopic anatomical parcellation of the MNI MRI single-subject brain. NeuroImage, 15, 273-289. doi:10.1006/nimg.2001.0978
Vorhold, V. (2008). The neuronal substrate of risky choice. Annals of the New York Academy of Sciences, 1128, 41-52. doi:10.1196/annals .1399 .006

Wager, T. D., Davidson, M. L., Hughes, B. L., Lindquist, M. A., \& OCHSNER, K. N. (2008). Prefrontal-subcortical pathways mediating successful emotion regulation. Neuron, 59, 1037-1050. doi:10.1016/ j.neuron.2008.09.006

Wellcome Trust Centre for Neuroimaging (2005). Statistical parametric mapping (SPM) [Software]. London: Wellcome Trust Centre for Neuroimaging.

Wetzels, R., Vandekerckhove, J., Tuerlinckx, F., \& WagenmakERS, E.-J. (2010). Bayesian parameter estimation in the Expectancy Valence model of the Iowa gambling task. Journal of Mathematical Psychology, 54, 14-27. doi:10.1016/j.jmp.2008.12.001

YATES, J. F. (ED.) (1992). Risk-taking behavior. Chichester, U.K.: Wiley.

\section{SUPPLEMENTAL MATERIALS}

The additional procedures and results referred to in this article may be downloaded from http://cabn.psychonomic-journals.org/content/ supplemental.

(Manuscript received October 7, 2009; revision accepted for publication April 12, 2010.) 